\title{
BMJ Open Health effects of training laypeople to deliver emergency care in underserviced populations: a systematic review protocol
}

\author{
Aaron M Orkin, ${ }^{1,2}$ Jeffrey D Curran, ${ }^{3}$ Melanie K Fortune, ${ }^{4}$ Allison McArthur, ${ }^{1}$ \\ Emma J Mew, ${ }^{1,3}$ Stephen D Ritchie, ${ }^{5,6}$ Stijn Van de Velde, ${ }^{7}$ David VanderBurgh ${ }^{3}$
}

To cite: Orkin AM, Curran JD, Fortune MK, et al. Health effects of training laypeople to deliver emergency care in underserviced populations: a systematic review protocol. BMJ Open 2016;6:e010609. doi:10.1136/bmjopen-2015010609

- Prepublication history and additional material is available. To view please visit the journal (http://dx.doi.org/ 10.1136/bmjopen-2015010609)

Received 22 November 2015 Revised 15 March 2016 Accepted 5 April 2016

CrossMark

For numbered affiliations see end of article.

Correspondence to

Dr Aaron M Orkin;

aaron.orkin@mail.utoronto.ca

\section{ABSTRACT}

Introduction: The Disease Control Priorities Project recommends emergency care training for laypersons in low-resource settings, but evidence for these interventions has not yet been systematically reviewed. This review will identify the individual and community health effects of educating laypeople to deliver prehospital emergency care interventions in lowresource settings.

Methods and analysis: This systematic review addresses the following question: in underserviced populations and low-resource settings $(P)$, does first aid or emergency care training or education for laypeople (I) confer any individual or community health benefit for emergency health conditions ( 0 ), in comparison with no training or other forms of education (C)? We restrict this review to studies reporting quantitatively measurable outcomes, and search 12 electronic bibliographic databases and grey literature sources. A team of expert content and methodology reviewers will conduct title and abstract screening and full-text review, using a custom-built online platform. Two investigators will independently extract methodological variables and outcomes related to patient-level morbidity and mortality and community-level effects on resilience or emergency care capacity. Two investigators will independently assess external validity, selection bias, performance bias, measurement bias, attrition bias and confounding. We will summarise the findings using a narrative approach to highlight similarities and differences between the gathered studies.

Ethics and dissemination: Formal ethical approval is not required.

Results: The results will be disseminated through a peer-reviewed publication and knowledge translation strategy.

Review registration number: CRD42014009685.

\section{INTRODUCTION}

Emergency care training for laypeople reduces morbidity and mortality in some resource-limited settings and in the

\section{Strengths and limitations of this study}

- First systematic review on individual and community health effects of resuscitative and nonresuscitative emergency care and first aid education programmes for laypeople in underserviced populations.

- The review protocol provides an exhaustive scholarly and grey literature search strategy, admits a wide variety of research methods and metrics, and offers highly inclusive definitions.

- Advances a novel, custom-developed web interface to engage an interdisciplinary review team of content and methodological experts, deliver reviewer training, and monitor quality metrics during screening.

- Will offer high level of evidence to understand the individual and community health effects of engaging laypeople in the delivery of emergency care and first aid.

- Anticipated heterogeneity of study types, study settings, interventions and reported outcomes are likely to hamper meta-analysis. A narrative review is expected.

management of specific health conditions such as physical trauma. ${ }^{1}{ }^{2}$ Laypeople, or bystanders with little or no training, may be an essential link in the 'chain-of-survival' from critical health emergencies. ${ }^{3}$ The effectiveness of emergency care training programmes may be enhanced in areas where laypeople are the sole providers of emergency care, or where existing formal health systems rely heavily on lay first responders to deliver care. $^{45}$

One systematic review demonstrated that lay providers and community health workers can improve health outcomes and reduce mortality for a variety of non-emergent health conditions including maternal child health and infectious disease. ${ }^{6}$ Reviews on non-resuscitative emergency care have 
demonstrated that first aid education increases helping behaviour, scores on first aid tests and simulation and confidence in administering first aid. ${ }^{7} 8$ A systematic review on task shifting for emergency care to nonphysician healthcare workers in Uganda identified potential benefits related to access to care and cost effectiveness, but did not specifically address laypeople in emergency care systems. ${ }^{9}$ A systematic review on trauma first aid concluded that interventions provided by laypeople show a potential to reduce trauma mortality. ${ }^{10}$ Bystander cardiopulmonary resuscitation (CPR) significantly improves out-of-hospital cardiac arrest survival, and the United States Institute of Medicine has called for a national public awareness and CPR training campaign. ${ }^{11} 12$ The International Liaison Committee on Resuscitation 2015 Consensus on First Aid Science provided a weak recommendation that education and training in first aid would improve morbidity and mortality from injury and illness. ${ }^{11} 13$

Guidelines and scientific reviews on first aid interventions are derived largely from studies based on inhospital practices or studies of emergency medical services. $^{7} 14$ The Disease Control Priorities Project recommends emergency care training for laypersons in low-resource settings, but the patient and community health effects of this training have not been reviewed systematically. ${ }^{15}$ Though first aid education remains a global and ubiquitous practice, it is unclear what emergency health conditions laypeople can treat effectively, what emergency interventions laypeople can deliver effectively, and in what settings and populations. The goal of this systematic review is to identify the individual and community health effects of training laypeople to deliver prehospital emergency care interventions in underserviced populations and low-resource settings.

\section{METHODS}

\section{Protocol registration and timeline}

This protocol is reported according to the Preferred Reporting Items for Systematic Reviews and MetaAnalyses extensions for systematic review protocols and reviews on health equity (PRISMA-P and PRISMA-E, see online supplementary appendix A). ${ }^{16}{ }^{17}$ Our team registered a standard version of the protocol through PROSPERO (the International Prospective Register of Systematic Reviews), and will manage necessary protocol amendments through this interface. (Protocol No. CRD42014009685). The Cochrane Handbook for Systematic Reviews of Interventions informs the methods described in this protocol. ${ }^{18}$

Preliminary searching and development of the search strategy occurred January to June 2014. Development of the complete protocol, review team and data management system occurred June 2014 to November 2015. Reviewer training, title and abstract review and full-text review is anticipated for February 2016-September 2016. Data extraction, analysis and manuscript preparation is anticipated to be completed by March 2017.

\section{Review question}

This systematic review addresses the following question: in underserviced populations and low-resource settings $(\mathrm{P})$, does first aid or emergency care training or education for laypeople (I) confer any individual or community health benefit for emergency health conditions $(\mathrm{O})$, in comparison with no training or other forms of education $(\mathrm{C})$ over any duration of time $(\mathrm{T})$ ?

- Population $(\mathrm{P})$ : Underserviced populations and populations in low-resource settings.

- Intervention (I): Any training or educational intervention regarding resuscitative and non-resuscitative first aid or emergency care delivered to laypeople.

- Control/comparator (C): Comparators may include no educational intervention, or the comparison of two different educational interventions. In the case of non-controlled studies, no comparator will be required.

- Outcome $(\mathrm{O})$ : Any individual or community health outcomes of these interventions, including any effects on morbidity, mortality, community capacity or community resilience. As such, individual and community health effects are defined broadly and holistically to favour any kind of measurable health outcome. These include primary outcomes related to physical or mental health, as well as secondary outcomes related to social indicators of health (ie, community capacity or resilience).

- Time (T): Studies of any duration, beginning after 1984.

\section{Definition of terms}

'Laypeople' refers to community members who have no professional certificate or health education degree. These individuals may have received training to promote health or to carry out some healthcare services. This definition aligns with terminology from previous systematic reviews on lay healthcare providers and non-resuscitative first aid. ${ }^{67}$ For the purpose of this review, 'laypeople' excludes paraprofessional cadres of healthcare workers such as community health workers. For example, a community health worker is not a layperson if employed primarily as a health worker, but a miner with first aid training is a layperson if employed primarily in mining but tasked with specialised occupational first aid duties. Providers with professional certifications who are employed to administer first aid are excluded from the layperson category, such as Registered Practical Nurses, Community Health Workers, Emergency Medical Technicians, even if they are not employed by a healthcare organisation. For example, an Emergency Medical Technician employed at a sporting event or mass gathering would not be a layperson.

Emergency care and first aid refer to the initial care of the acute manifestation of any illness or injury where the time from problem identification to treatment should occur within minutes or hours to reduce suffering, morbidity or mortality. ${ }^{19}$ This includes, but is not 
limited to, injuries and environmental exposures, myocardial infarction, stroke and acute manifestations of chronic diseases such as diabetes, mental health disorders or substance misuse. Emergency care provided by laypeople may be definitive or may result in the transfer of care to professional clinicians. When provided by laypeople, emergency care includes but is not limited to first aid interventions as defined by the American Heart Association and American Red Cross, the European Resuscitation Council, or by other international first aid guidelines. ${ }^{19} 2021$ Our definition of emergency care is therefore comparatively broader than those used for first aid guidelines and consensus statements. ${ }^{14}$ Emergency care and first aid does not include intrapartum or neonatal care because previous reviews have explored lay midwifery, lay birth attendants and doulas, and because routine pregnancy or birthing is not considered an illness. ${ }^{6}$

Underserviced populations or low-resource settings refer to groups of people who face barriers to accessing prehospital emergency services. Barriers may include geographical, financial, occupational, sociopolitical, racial and ethnocultural, infrastructural or informational factors. Based on this definition, these populations include, but are not limited to, rural or remote populations, occupational settings with specific needs, low and middle income countries, or populations in wellresourced settings that face access barriers to otherwise developed services. ${ }^{22-24}$ These definitions capture all populations included in a previous Cochrane review on lay providers in the delivery of maternity care services in low-resource settings, but are substantially broader due to the inclusion of individuals who may reside in wellresourced settings but face other barriers to accessing emergency care. ${ }^{6}$

Educational programmes are initiatives designed to confer knowledge or change attitudes and behaviours about identifying or responding to emergency health conditions. Educational programmes include but are not limited to first aid skills training programmes known as first aid or first responder training courses, but our definition is more inclusive to allow for any form of emergency care education. Educational programmes may use any teaching and learning modality or tools (eg, written, electronic, verbal, experiential, observership, simulation, scenario etc), and may be targeted or more widely disseminated (eg, a programme that delivers workplace anaphylaxis education to school teachers, or a public poster campaign about first aid for choking).

\section{Types of study}

We restrict this review to studies reporting quantitatively measurable outcomes and will exclude purely qualitative studies. Few emergency care education initiatives in lowresource settings have been studied using randomised controlled trials (RCTs). For this reason, we will include studies and programme evaluations based on fit-for-purpose, and are not limiting the scope to RCTs. The following study types are eligible for inclusion: RCTs and variants, observational studies (case control studies, cohort studies, cross-sectional studies), quasiexperimental designs, case series studies, programme evaluations and quality improvement studies.

\section{Date and language restrictions}

We will include scientific studies published within the past 30 years (1984-2014) in the search to reduce the yield to a feasible volume. Following the initial review and analysis, we will update the search to the present date to capture recent publications. Language of publication does not restrict inclusion, though English search terms were used to identify studies. Where English search terms yield study information in languages other than English, French, Dutch, Norwegian and German, we will translate the title and abstract using Google Translate for title and abstract review, full-text review, data extraction and risk of bias assessment. ${ }^{25}$

Table 1 summarises the study inclusion and exclusion criteria.

\section{SEARCH}

In accordance with best practices in systematic review designs, we executed informational and search strategies in collaboration with a medical and public health information scientist $(\mathrm{AM}) .^{26-28}$

Table 1 Inclusion and exclusion criteria for systematic review

\begin{tabular}{ll}
\hline Inclusion criteria & Exclusion criteria \\
Quantitative measure of health outcome & No quantitative \\
Any emergency health condition & assessment \\
Analysis concerns people who face barriers to accessing conventional prehospital emergency & No acute health \\
services (full study population or subgroup) & condition \\
Educational intervention regarding resuscitative or non-resuscitative first aid or emergency care & No underserviced \\
Target population for the educational intervention has no formal professional certificate or & population \\
health education degree, is not employed primarily in the delivery of healthcare, and/or is & No laypeople \\
performing first aid as a citizen rather than an organisational employee or volunteer & No educational \\
representative & Published before 1984
\end{tabular}




\section{Data base search}

We searched the following electronic bibliographic databases MEDLINE, Embase, Cumulative Index to Nursing and Allied Health Literature (CINAHL), Scopus, SocINDEX, PsycINFO, Educational Resources Information Center (ERIC), Cochrane Database of Systematic Reviews, African Index Medicus (AIM), Index Medicus for the WHO Eastern Mediterranean Region (IMEMR), Latin American and Caribbean Center on Health Sciences Information (LILACS), Index Medicus for South-East Asia Region (IMSEAR) and Transport Research International Documentation (TRID).

We harvested search terms from known literature reviews on layperson training interventions and articles identified by snowball searches using PubMed's 'Related Articles' feature. ${ }^{6}$ We used these terms to develop a preliminary MEDLINE search strategy to find articles on prehospital emergency care education interventions for laypeople. We screened the results of this preliminary search to identify studies for inclusion in a representative sample set of relevant articles for search term relevancy evaluation. We used this set to test the precision and recall of terms and inform the final selection of search terms.

We developed a two-concept search strategy designed to retrieve articles containing at least one search term (controlled term or keyword) from the 'prehospital care' and 'layperson' concepts. We searched keywords in the title, abstract and 'keyword heading' fields where available.

We selected search terms for prehospital care for strategy inclusion based on treatment intervention (first aid, $\mathrm{CPR}$, etc) rather than health condition (frostbite, myocardial infarction, etc). Search terms for specific health conditions were tested but not included because they returned many false hits without retrieving new unique articles. The low specificity of health condition terms was due, in part, to some conditions only being classified as emergencies when they become severe (eg, wounds and injuries), and because these terms retrieve many false hits on inhospital care for health conditions by trained laypeople when not combined with terms related to emergencies.

Neither the medical subject heading hierarchy nor the controlled vocabularies of the other health and social science databases captured the layperson concept. We developed an essentiality assessment process to evaluate recall and precision of all search terms within the laypeople concept. ${ }^{29}$ We considered a search term essential if it retrieved known relevant articles that could not be found using any other synonym through an iterative search process. We repeated the essentiality assessment process for all terms in the layperson concept, and incorporated essential search terms with high recall and low precision into the strategy to ensure comprehensive retrieval.

We adapted the final MEDLINE search strategy (see online supplementary appendix B) to leverage the subject headings available in each database and ensure compatibility with the search operators of each database platform and grey literature search tool. Search adaptations favoured sensitivity over specificity where advanced search operators were unavailable.

\section{Grey literature search}

The contribution of grey literature is especially relevant because layperson interventions involve community members and organisations. The International Liaison Committee on Resuscitation and other scientific review panels for first aid guidelines have not included grey literature search strategies or evidence arising from nonacademic sources. ${ }^{8} 101422$ We developed a robust grey literature search strategy using a unique keyword-based strategy for each searchable grey literature source.

We conducted grey literature searches using general web searches (Google), custom web searches, targeted web searches (eg, WHO, Canadian Red Cross, UNICEF, Médecins Sans Frontières), grey literature repositories (OpenGrey, New York Academy of Medicine Grey Literature Report and Canadian Best Practices Portal), theses and dissertations (ProQuest Dissertations \& Theses Database), clinical trials registries (International Clinical Trials Registry Platform), and proceedings of relevant conferences.

We scaled the complexity of each search strategy to the search functionality of each source to leverage each platform and promote comprehensive retrieval. We executed multiple shorter queries when search query word limits were restrictive. Where search interfaces such as Google made it impossible to know with certainty how queries were interpreted by the search engine, we prioritised search terms based on recall/precision during essentiality assessment, and developed 20 separate queries which combined the most useful terms from the two search concepts. We made the queries as sensitive as possible within the search engine's 32-word query limit. We retrieved the first 50 results for each web search query for review. We retrieved all results for review for all remaining grey literature sources.

\section{Snowballing and citation search}

We used two snowballing techniques. First, we tracked citations of all included scientific and grey literature to capture cited studies not identified through database searching. Second, we manually searched citations of existing first aid guidelines and evidence reviews from the American Heart Association and Red Cross, International Liaison Committee on Resuscitation and European Resuscitation Council. ${ }^{14} 2021$

We will update the MEDLINE search following the study selection process and analysis, and will review studies between 2014 and submission using the same eligibility criteria. The lead investigators will incorporate new studies into the review. 


\section{Reviewers and reviewer training}

We recruited a diverse interdisciplinary review team including nurses, physicians, paramedics and researchers. Reviewers consist of both content experts in emergency care and task shifting, as well as non-content experts. Where possible, content experts will be paired with non-content experts in title and abstract review to address underlying assumptions and preconceived notions that the content experts may have regarding the merit of included studies.

We created a custom-built website and data management system for screening using Google Sites, which offered a system to manage and share up-to-date information between investigators and reviewers. ${ }^{30}$ This platform houses the reviewer training materials and review sets, review protocol, project timeline, frequently asked questions and announcement board. The platform enables customisation for each reviewer to have access to materials, while the investigators can access all training sets and reviewer batches.

We will train reviewers using a purpose-developed internet video before screening a training set of 70 citations. The training set consists of a random subset of the search results identified for selection with the addition of seven key studies meeting some or all inclusion criteria as determined by lead investigators. All reviewers will complete the training set and we will calculate the inter-rater reliability (Fleiss' $\kappa$ ) to evaluate the reviewers' training set performance. The investigators will discuss reviewer questions in an open online forum. Reviewers will require substantial inter-rater agreement to pass the training and subsequently move on to title and abstract review (Fleiss' $\kappa$ score $>0.61$ ) ${ }^{31}$ If we do not achieve substantial agreement between reviewers, we will conduct additional training or further specify inclusion and exclusion criteria.

\section{Study selection}

We will identify studies which meet the inclusion criteria using a two-stage approach: (1) title and abstract screening, and (2) full-text screening.

\section{Title/abstract screening}

Two reviewers will independently screen titles and abstracts, using the parameters in table 1. Citations will be batched into groups of $\sim 1250$. Each batch contains a proportional amount of citations drawn from each database in the literature search. Each reviewer will screen the titles and abstracts of at least one batch and will only gain access to their batches. We will address discrepancies through discussion with a third reviewer and additional training based on eligibility criteria. If after discussion there is still doubt concerning study inclusion, we will retain it for full-text screening.

Reviewers are not blinded to the origin or authorship of the primary studies, and will be encouraged to search the abstract online for missing information. Masking the origin of primary studies is not necessary and may not improve the quality of review. ${ }^{32}$ Maintaining origin and authorship of studies is especially relevant because noncontent expert reviewers may have less experience with the literature.

\section{Full-text screening}

Two expert reviewers from the authorship team will independently assess full-text copies of studies included from title and abstract screening. A discussion between the two reviewers and a lead investigator will resolve discrepancies. We will report excluded studies at this stage in a 'Characteristics of Excluded Studies' table.

We will link multiple reports of the same study through examination of study details. Where necessary, we will correspond with study authors to clarify study eligibility, and request further information. Included studies will move on to data collection.

\section{DATA COLLECTION}

Two investigators will independently extract data from the included studies using an electronic data collection form. All data extractors will be familiar with the data collection form prior to extracting information from the included studies, and the form will be piloted using a sample of studies. Investigator feedback may modify the data collection form based on coding instructions that are confusing or incomplete, or that contain missing or unnecessary data. Where multiple reports exist on a single study, we will extract data from all reports into a single data collection form with each citation listed. The lead investigators will check the information gathered for irregularities and will remedy discrepancies through discussion. We will record discrepancies and will synthesise recorded data from each investigator into a consensus data form.

\section{Data items}

We will extract the following from included studies: information regarding the study (citation, design, objective), population (size, age, sex, geographic location, setting, reason for underserviced status, sociodemographics), intervention (mode of education, length of training, emergency health condition, layperson role, cost), control group (existence, size, geographic location, setting, reason for underserviced status), outcome (type of health outcome, description of health outcome, type of emergency care provided, effect size and confidence interval reported), and key conclusions of the study authors. We will collect primary outcomes regarding any individual or community health outcome related to physical or mental health. We will also collect secondary quantitative outcomes related to any social indicators of health at the individual or community level, such as metrics of community capacity or community resilience, and indicators of trained laypeople providing emergency care. 
Risk of bias in individual studies

We will assess the following quality constructs for each study: external validity, selection bias, performance bias, measurement bias, attrition bias and confounding. We will accomplish this using standardised quality appraisal tools relevant to each study type such as the Downs and Black checklist and the Effective Public Health Practice Project quality assessment tool. ${ }^{33} 34$

Two investigators will assess risk of bias independently. We will resolve disagreements in consultation with a third investigator. Risk of bias tables will summarise the quality of individual studies.

\section{SUMIMARY MEASURES}

We will compile the number of reports and corresponding studies along with appropriate summary measures for each health effect, such as differences in morbidity, mortality, community resilience or capacity measures. We will also calculate mean differences, where possible, for studies with continuous data and the relative risks for studies with dichotomous data.

\section{Synthesis of results}

We will summarise the included studies' characteristics and findings using a narrative approach because of the anticipated heterogeneity of studies, and will describe the intervention effects, reasons for any observed heterogeneity and the robustness of findings given identified biases. We will structure this narrative synthesis of results to highlight similarities and differences between studies. If there are a sufficient number of adequately similar studies, we will update our protocol with a statistical analysis plan through PROSPERO before conducting a random effects meta-analysis on aggregate data to estimate an overall effect size.

We will group studies that compare similar types of interventions or emergency health problems. Within each group, we will describe the setting, population, intervention and outcome using a narrative approach. A 'Summary of Findings' table will illustrate the available studies and weight of evidence for each intervention. We may undertake subgroup analyses for exploratory purposes depending on the volume and variability of the included studies. While specific subgroups cannot be anticipated in advance, if used, they would be based on characteristics of the populations, interventions and outcomes of the studies.

\section{Risk of bias across studies}

We will assess the collective risk of bias using a standardised tool, and this will influence the conclusions and recommendations following data synthesis. We will use the GRADE approach (Grading of Recommendations, Assessment, Development and Evaluation) to assess the quality of the body of evidence for each individual outcome. ${ }^{35}$ We will consider results based on randomised trials as higher quality than observational studies (eg, cohort, case-control, cross-sectional, case series), however, we will evaluate each study on its own metrics and the culmination of such quality assessments considered in the collective body of evidence. We will address the risk of publication bias through the search process in scanning grey literature and conference proceedings. If meta-analysis is possible, we will also develop a funnel plot to assess publication bias.

\section{ETHICS AND DISSEMINATION}

This systematic review does not involve primary data collection, does not constitute research on human participants, and is not subject to additional ethical review or informed consent procedures. Results will be disseminated through a knowledge translation strategy involving an open-access peer-reviewed publication, academic conference presentations, and correspondence with stakeholders such as first aid training and guidelines organisations and researchers.

\section{CONCLUSION}

Though first aid is a globally ubiquitous practice, and task shifting to laypeople may address barriers to accessing essential care, little is known about the measurable health effects of first aid training and education in lowresource settings. This review will provide a summary of scientific knowledge on the measurable health effects of laypeople, and first aid training and education in the delivery of emergency care in low-resource settings. The review protocol provides an exhaustive scholarly and grey literature search strategy, admits a wide variety of research methods and metrics, and offers a highly inclusive definition of underserved populations and individual and community health effects. Overall, the review will provide a first summary of existing science on the role of laypeople in the delivery of emergency care in low-resource settings.

\section{Author affiliations}

${ }^{1}$ Dalla Lana School of Public Health, University of Toronto, Toronto, Ontario, Canada

${ }^{2}$ Rescu, Li Ka Shing Knowledge Institute, St. Michael's Hospital, Toronto, Ontario, Canada

${ }^{3}$ Northern Ontario School of Medicine, Thunder Bay, Ontario, Canada ${ }^{4}$ Michael G. DeGroote School of Medicine, McMaster University, Hamilton, Ontario, Canada

${ }^{5}$ Laurentian University, Sudbury, Ontario, Canada

${ }^{6}$ Centre for Rural and Northern Health Research, Sudbury, Ontario, Canada

${ }^{7}$ Academic Centre for General Practice, Katholieke Universiteit Leuven, Leuven, Belgium

Contributors AMO, DV, SDR and SVV developed the study concept and question. AMO and DV provided overall project leadership and management. JDC, AM and AMO developed the initial draft of the protocol, and all authors reviewed and refined the protocol manuscript. AM, DV, AMO and MKF developed and executed the search strategy. MKF, JDC and AMO developed and revised the protocol for PROSPERO registration. EJM and JDC developed the online review interface and reviewer training materials and managed manuscript submission. 
Funding This project received financial support from the Northern Ontario Academic Medicine Association Innovation Fund (Project \#A-15-07).

Competing interests AMO and DV declare a non-financial conflict of interest through their affiliation with the Remote Health Initiative, a non-profit entity dedicated to enhancing care in remote settings. SVV declares an intellectual conflict of interest through his authorship of the African First Aid Guideline.

Provenance and peer review Not commissioned; externally peer reviewed.

Open Access This is an Open Access article distributed in accordance with the Creative Commons Attribution Non Commercial (CC BY-NC 4.0) license, which permits others to distribute, remix, adapt, build upon this work noncommercially, and license their derivative works on different terms, provided the original work is properly cited and the use is non-commercial. See: http:// creativecommons.org/licenses/by-nc/4.0/

\section{REFERENCES}

1. Murad MK, Husum $\mathrm{H}$. Trained lay first responders reduce trauma mortality: a controlled study of rural trauma in Iraq. Prehosp Disaster Med 2010;25:533-9.

2. Jayaraman S, Mabweijano JR, Lipnick MS, et al. First things first: effectiveness and scalability of a basic prehospital trauma care program for lay first-responders in Kampala, Uganda. PLOS ONE 2009;4:e6955.

3. Cummins RO. The "chain of survival" concept: how it can save lives. Heart Dis Stroke 1991;1:43-5.

4. Kobusingye OC, Hyder AA, Bishai D, et al. Emergency medical systems in low-and middle-income countries: recommendations for action. Bull World Health Organ 2005;83:626-31.

5. Bhanji F, Donoghue AJ, Wolff MS, et al. Part 14: education: 2015 American Heart Association guidelines update for cardiopulmonary resuscitation and emergency cardiovascular care. Circulation 2015;132:S561-73

6. Lewin S, Munabi-Babigumira S, Glenton C, et al. Lay health workers in primary and community health care for maternal and child health and the management of infectious diseases. Cochrane Database Syst Rev 2010;(3):CD004015.

7. Van de Velde S, Heselmans A, Roex A, et al. Effectiveness of nonresuscitative first aid training in laypersons: a systematic review. Ann Emerg Med 2009;54:447-57.

8. He Z, Wynn P, Kendrick D. Non-resuscitative first aid training for children and laypeople: a systematic review. Emerg Med $J$ 2014;31:763-8.

9. Terry B, Bisanzo M, McNamara M, et al. Task shifting: Meeting the human resources needs for acute and emergency care in Africa. Afr J Emerg Med 2012;2:182-7.

10. Tannvik TD, Bakke HK, Wisborg T. A systematic literature review on first aid provided by laypeople to trauma victims. Acta Anaesthesiol Scand 2012;56:1222-7.

11. Sasson C, Rogers MAM, Dahl J, et al. Predictors of survival from out-of-hospital cardiac arrest: a systematic review and meta-analysis. Circ Cardiovasc Qual Outcomes 2009;3:63-81.

12. Graham R, McCoy MA, Schultz AM, eds., Committee on the Treatment of Cardiac Arrest: Current Status and Future Directions; Board on Health Sciences Policy; Institute of Medicine. Strategies to improve cardiac arrest survival: a time to act. Washington, DC: National Academies PressUS, 2015.

13. American Heart Association, American Stroke Association, International Liaison Community on Resuscitation. First Aid Training 2015. https://volunteer.heart.org/apps/pico/Pages/PublicComment. aspx?q=773 (accessed Nov 2015).
14. Singletary EM, Charlton NP, Epstein JL, et al. Part 15: First Aid: 2015 American Heart Association and American Red Cross Guidelines Update for First Aid. Circulation 2015;132Suppl 2):S574-89.

15. Kobusingye OC, Hyder AA, Bishai D, et al. Chapter 68: emergency medical services. In: Jamison DT, Breman JG, Measham AR, et al. Disease control priorities in developing countries. 2nd edn. Washington DC: The World Bank and Oxford University Press, 2006:1261-79.

16. Moher D, Shamseer L, Clarke M, et al. Preferred reporting items for systematic review and meta-analysis protocols (PRISMA-P) 2015 statement. Syst Rev 2015;4:1.

17. Welch V, Petticrew M, Tugwell $P$, et al. PRISMA equity 2012 extension: reporting guidelines for systematic reviews with a focus on health equity. PLoS Med 2012;9:e1001333.

18. Higgins JPT, Green S. Cochrane handbook for systematic reviews of interventions, version 5.1.0. The Cochrane Collaboration, 2011. http://www.cochrane-handbook.org

19. Markenson D, Ferguson JD, Chameides L, et al. Part 17: First Aid 2010 American Heart Association and American Red Cross Guidelines for First Aid. Circulation 2010;122(Suppl 3):S934-46.

20. Van de Velde S, Broos $\mathrm{P}$, Van Bouwelen M, et al European First Aid Manual Project, Belgian Red Cross-Flanders. European first aid guidelines. Resuscitation 2007;72:240-51.

21. Van de Velde S, De Buck E, Vandekerckhove $\mathrm{P}$, et al. Evidence-based African first aid guidelines and training materials. PLoS Med 2011;8:e1001059.

22. UyBico SJ, Pavel S, Gross CP, et al. Recruiting vulnerable populations into research: a systematic review of recruitment interventions. J Gen Intern Med 2007;22:852-63.

23. Flaskerud $\mathrm{JH}$, Winslow BJ. Conceptualizing vulnerable populations health-related research. Nurs Res 1998;47:69-78.

24. Weitz TA, Freund KM, Wright L. Identifying and caring for underserved populations: experience of The National Centers of Excellence in Women's Health. J Womens Health Gend Based Med 2001;10:937-52.

25. Google. Google Translate. https://translate.google.ca (accessed Nov 2015).

26. Alpi KM. Expert searching in public health. J Med Lib Assoc 2005;93:97-103.

27. National Academy of Science. Standards for systematic review. 2015. http://www.iom.edu/Reports/2011/Finding-What-Works-inHealth-Care-Standards-for-Systematic-Reviews/Standards.aspx? page $=2$ (accessed Nov 2015).

28. Relevo R, Balshem $\mathrm{H}$. Finding evidence for comparing medical interventions: AHRQ and the Effective Health Care Program. J Clin Epidemiol 2011;64:1168-77.

29. Almanssori S. Creating a comprehensive search strategy for research on learning disabilities using the pearl harvesting information retrieval framework (dissertation). London, Ontario: University of Western Ontario, 2015:49.

30. Google. Google sites overview. 2011. http://www.google.com/sites/ overview.html (accessed Nov 2015).

31. Landis JR, Koch GG. The measurement of observer agreement for categorical data. Biometrics 1977;33:159-74.

32. Berlin JA. Does blinding of readers affect the results of meta-analyses? Lancet 1997;350:185-6.

33. Downs SH, Black N. The feasibility of creating a checklist for the assessment of the methodological quality both of randomised and non-randomised studies of health care interventions. J Epidemiol Community Health 1998;52:377-84.

34. Effective Public Health Practice Project. Quality assessment tool for quantitative studies. http://www.ephpp.ca/PDF/ Quality20Assessment20Tool 2010 2.pdf (accessed Nov 2015).

35. Guyatt $\mathrm{GH}$, Oxman AD, Schünemann HJ, et al. GRADE guidelines: a new series of articles in the Journal of Clinical Epidemiology. J Clin Epidemiol 2011;64:380-2. 